\title{
Fortalecimiento de las características emprendedoras personales en estudiantes de Química Analítica I y II
}

\author{
Noelia Centurión \\ ncenturion@qui.una.py
}

Amapola Cabrera

acabrera@qui.una.py

Celso Mora

cmora@qui.una.py

Programa de Apoyo a Investigación Educativa de la Facultad de Ciencias Químicas de la Universidad Nacional de Asunción

\section{RESUMEN}

El emprendimiento y la innovación están unidos al crecimiento económico y a la renovación industrial, por lo que el desarrollo de una cultura emprendedora y las competencias asociadas a emprender necesita una mayor atención de parte de la educación formal. El objetivo de esta investigación se centró en la evaluación del fortalecimiento de las características emprendedoras personales en los estudiantes proporcionado a través del Aprendizaje Basado en Problemas y el Aprendizaje Colaborativo en los Laboratorios de Química Analítica I y Química Analítica II de las Carreras de Licenciatura en Ciencia y Tecnología de Alimentos y Licenciatura en Química Industrial. Los datos fueron obtenidos mediante la utilización del Cuestionario de Autoevaluación de las Características Emprendedoras Personales entregado a los estudiantes al inicio y a fin de año, de modo a medir la influencia de las metodologías de enseñanza sobre las mismas a través de un análisis paramétrico para muestras relacionadas, arrojando el resultado de que solo la Autoconfianza e Independencia demostró una diferencia significativa mientras que las restantes nueve características no sufrieron un cambio. Por lo que se concluyó que la modificación en la planificación aplicada no fue suficiente como para observar el aumento de las características emprendedoras personales.

Palabras clave: características emprendedoras personales; aprendizaje basado en problemas; aprendizaje colaborativo; clases prácticas. 


\title{
Strengthening of personal entrepreneurships characteristics in the laboratory students of analytical chemistry I and analytical chemistry II
}

\begin{abstract}
Entrepreneurship and innovation are linked to economic growth and industrial renewal, so the development of an entrepreneurial culture and the skills associated with entrepreneurship requires greater attention by formal education. The objective of this research focused on the evaluation of the strengthening of the Personal Entrepreneurship's Characteristics in students provided through Problem Based Learning and Collaborative Learning in the laboratories of Analytical Chemistry I and Analytical Chemistry II of the Degree in Food Science and Technology and Chemical Industry. The data was obtained sing the Self-Assessment Questionnaire of Personal Entrepreneurships Characteristics delivered to students at the beginning and at the end of the year, in order to measure the influence of teaching methodologies on them through a parametric analysis for related samples, yielding the result that only Self-Confidence and Independence demonstrated a significant difference while the remaining nine characteristics did not undergo a change. Therefore, it was concluded that the modification of the learning planning was not enough to observe the increase of the personal entrepreneurship's characteristics.
\end{abstract}

Keywords: personal entrepreneurship's characteristics; problem based learning; collaborative learning;

Artículo recibido: 02 Setiembre. 2021 Aceptado para publicación: 30 Setiembre. 2021

Correspondencia: ncenturion@qui.una.py Conflictos de Interés: Ninguna que declarar 


\section{INTRODUCCIÓN}

En el momento de la planificación de las clases, en general, el docente universitario se asegura de que el contenido del programa de estudios pueda ser desarrollado íntegramente, teniendo el objetivo central de que el estudiante pueda culminar la asignatura con nuevos conocimientos, tanto teóricos como prácticos, los cuales son necesarios para la vida profesional. En este sentido, en las clases prácticas del laboratorio, los estudiantes ingresan al aula, de manera automática, considerando de antemano que ya cuentan con el guía que debe ser seguido al pie de la letra para lograr el objetivo de este. Pero, en el instante que se presenta alguna dificultad, recurren al docente en busca de la solución sin haber invertido un tiempo o recursos personales para resolver el problema. Esta situación ocurre debido a que el aprendizaje no fue realizado en su totalidad, ya que el mismo se trata de un proceso de construcción personal del saber, saber hacer y saber ser para querer hacer y muchas veces el mayor esfuerzo del profesor está enfocado en los conceptos y procedimientos obviando el dominio actitudinal, y por ende, el estudiante tampoco no lo considera trascendental, siendo valorado por el mismo recién una vez que se encuentra en el ambiente laboral, teniendo dificultades en las relaciones interpersonales, careciendo de las respuestas a cada situación o inclusive encontrándose desempleado sin saber qué hacer.

En el documento final de las Metas Educativas 2021, La Educación que queremos para la generación de los Bicentenarios, aprobado por la XX Cumbre Iberoamericana de Jefes de Estado y Gobierno, la meta general sexta propone «favorecer la conexión entre la educación y el empleo» promoviendo el mejoramiento y la adaptación de los diseños curriculares de la educación técnico-profesional y el aumento de los niveles de inserción laboral de la población joven egresada de la educación técnica profesional (OEI, 2010) En este contexto, el propósito principal de la OEI es colaborar con todos los países miembros en el desarrollo de políticas de reforma de la formación profesional que contribuyan a su perfeccionamiento y a incorporar, en la cultura de los jóvenes y de los trabajadores, la formación emprendedora y el aprendizaje a lo largo de toda la vida para mejorar sus posibilidades de obtener un empleo. Es preciso que la enseñanza se oriente hacia la innovación, el trabajo en equipo, los proyectos colectivos y el cuidado de las dimensiones emocionales y personales que dan seguridad para enfrentarse a nuevos desafíos. 
Al hacer una revisión de las competencias que se espera posean los egresados de las Licenciaturas de Ciencia y Tecnología de Alimentos (FCQ UNA, Facultad de Ciencias Químicas, 2020) y Química Industrial (FCQ UNA, Facultad de Ciencias Químicas, 2020) una vez finalizada la carrera, se encuentra que los mismos deben ser capaces de realizar no solo análisis químicos, que controlen la materia prima, el proceso y el producto, sino que también deben ser capaces de dirigir, supervisar, formar parte de equipos de trabajo, investigar e innovar en diferentes campos de estudio, llevar adelante regencias y consultorías, entre otras más, por lo que se podría englobar que se busca que los licenciados también puedan ser emprendedores en su profesión. Ambas carreras no poseen en la malla curricular una asignatura que les fortalezca las características emprendedoras personales, por lo que es importante que la planificación de las asignaturas ya existentes enfatice dichas actitudes.

La adquisición efectiva de las numerosas competencias que definen cada titulación requiere que el alumno aprenda haciendo. Resultaría imposible garantizar que nuestros alumnos aprendan a comunicar si en nuestras enseñanzas no hay espacio para que ellos expongan trabajos o elaboren informes. No aprenderán a planificarse si sólo planificamos nosotros. No aprenderán a seleccionar, manejar e integrar la información si nunca consultan otras fuentes que no sean nuestros apuntes o un libro de texto.

La metodología del Aprendizaje Basado en Problemas (ABP) es una colección de situaciones problemáticas cuidadosamente construidas para ser presentadas a pequeños grupos de estudiantes auxiliados por un tutor. Los problemas, generalmente, consisten en una descripción en lenguaje muy sencillo y poco técnico de conjuntos de hechos o fenómenos observables que plantean un reto o una cuestión, es decir, requieren explicación. La tarea del grupo de estudiantes es discutir estos problemas y producir explicaciones tentativas para los fenómenos describiéndolos en términos fundados de procesos, principios o mecanismos relevantes (Norman \& Schmidt, 1992).

El Aprendizaje Colaborativo (AC) es una técnica didáctica que promueve el aprendizaje centrado en el alumno basando el trabajo en pequeños grupos, donde los estudiantes con diferentes niveles de habilidad utilizan una variedad de actividades de aprendizaje para mejorar su entendimiento sobre una materia. Cada miembro del grupo de trabajo es responsable no solo de su aprendizaje, sino de ayudar a sus compañeros a aprender, 
creando con ello una atmósfera de logro. Los estudiantes trabajan en una tarea hasta que los miembros del grupo la han completado exitosamente (TEC de Monterrey).

Ser emprendedor supone un conjunto de competencias que les hace poseedores de un perfil diferente al resto de las personas ya que cuentan con las capacidades de detectar oportunidades con creatividad y resolución, rompiendo con ideas previas, para crear nuevas formas de hacer las mismas cosas; percibir novedades, detalles, signos, símbolos, coyunturas, momentos, ajustes y compromisos, aunque pasen desapercibidos para otros; asumir riesgos, y enfrentarlos con la confianza de poder vencerlos; poseer un elevado nivel de interés por la aventura y el empeño por descubrir nuevos métodos y encontrar salidas diferentes a los problemas habituales; creer que el cambio es posible y trabajan en ello: son creativos, creadores y resolutivos; acometer un proyecto que es rechazado por la mayoría y sabe interpretar las características reales del entorno a pesar de que no son aparentes a su competencia; luchar ante cualquier inconveniente que se interponga en su estrategia y no le teme al fracaso; crear un grupo con la motivación que le da la estructura requerida y enfrentar eficazmente los desafíos que presenta el cambio constante, satisfacer a los clientes por encima de lo que la competencia ofrece y promover un enriquecimiento en el que todos ganan (FAUTAPO, 2010).

Fundamentado en la Teoría de las Necesidades, McClelland realizó estudios con empresarios a nivel mundial y obtuvo resultados de comportamientos que caracterizaban a los gerentes como personas organizadas, ordenadas, planificadoras, arriesgadas, inteligentes, claras en cuanto a lo que buscan, creativas, líderes, investigadoras, insistentes, con buenas relaciones con los demás, etc., resumiendo toda la información recolectada en el modelo de 10 Características Emprendedoras Personales (CEP's) que consisten en:

- Búsqueda de Oportunidades e Iniciativa

- Persistencia

- Cumplimiento de Compromisos

- Exigencia de Eficiencia y Calidad

- Asumir Riesgos

- Fijación de metas

- Búsqueda de Información

- Planificación sistemática y seguimiento 
- Persuasión y construcción de redes de apoyo

- Autoconfianza e Independencia. (Briasco, 2014).

Las Clases Prácticas de Laboratorio consisten en una modalidad organizativa en la que se desarrollan actividades de aplicación de los conocimientos a situaciones concretas y de adquisición de habilidades básicas y procedimentales relacionadas con la materia, siendo las competencias por adquirir dependientes del entorno físico y los recursos disponibles. El esquema de las actividades de las prácticas del laboratorio permite que el estudiante tenga un contacto más cercano con el docente por lo que, si el mismo está consciente de todo lo que puede influir en el alumno, puede valorar el alcance de cada actividad planificada buscando el crecimiento en todas las dimensiones de la persona.

El siguiente trabajo se centró en el análisis del fortalecimiento de las Características Emprendedoras Personales a través de las clases prácticas de laboratorio de Química Analítica I y Química Analítica II, teniendo cada una sus particularidades en cuanto al desarrollo de las actividades, mediante la aplicación del Cuestionario de Autoevaluación.

\section{ESTRATEGIAS METODOLÓGICAS O MATERIALES Y MÉTODOS}

Este trabajo de investigación fue realizado en la Facultad de Ciencias Químicas de la Universidad Nacional de Asunción, específicamente en estudiantes que cursaron en el 2017 los Laboratorios de Química Analítica I (QAI) y Química Analítica II (QAII) del Segundo y Tercer Nivel respectivamente, de las carreras de Licenciatura de Ciencia y Tecnología de Alimentos y Licenciatura en Química Industrial.

La investigación realizada posee un enfoque cuantitativo con alcance descriptivo.

La recolección de datos se realizó utilizando el Cuestionario de Características Emprendedoras Personales (Briasco, 2014), instrumento utilizado internacionalmente para la valoración y su posterior análisis estadístico. El estudio descriptivo busca especificar las propiedades, las características y los perfiles de los estudiantes, mostrando los ángulos o dimensiones de un fenómeno.

La población se encuentra compuesta por 29 estudiantes de la Carrera de Licenciatura en Ciencia y Tecnología de Alimentos y 22 estudiantes de la Carrera de Licenciatura en Química Industrial.

Los mismos son alumnos que en el primer semestre cursan el Laboratorio de Química Analítica I (segundo nivel) y en el segundo semestre, el Laboratorio de Química Analítica II (tercer nivel). 
A través de la evaluación del instrumento de recolección de datos, las variables dependientes a ser analizadas son las 10 características emprendedoras personales.

\subsection{Fases de la Elaboración del Trabajo de Investigación.}

\subsubsection{Medición Inicial de las Características Emprendedoras Personales.}

Al comienzo del Primer Semestre del 2017, en el que los estudiantes cursaban la asignatura de Química Analítica I, antes del inicio de las prácticas de laboratorio se les entregó, de manera individual el cuestionario que se compone por 55 declaraciones breves. Los alumnos debían leer cuidadosamente cada declaración y decidir la frecuencia que le representa, en forma más acertada, siendo honestos, seleccionando el número que corresponde para designar la medida en que la declaración le describe de acuerdo con la

Tabla 2-1. Puntuación para el listado de las declaraciones

\begin{tabular}{|l|c|}
\hline \multicolumn{1}{|c|}{ Frecuencia } & Puntuación \\
\hline Nunca & 1 \\
\hline Raras veces & 2 \\
\hline Algunas Veces & 3 \\
\hline Usualmente & 4 \\
\hline Siempre & 5 \\
\hline
\end{tabular}

El listado de declaraciones se evalúa agrupándolas por cada característica, como indican las Tablas 2-2 a la 2-11.

Tabla 2-1 Declaraciones que evalúan la Búsqueda de Oportunidades e Iniciativa

\begin{tabular}{|l|l|}
\hline D1 & Me esmero en buscar cosas que necesitan hacerse. \\
\hline D2 & Hago lo que se necesita hacer sin que otras personas me lo pidan. \\
\hline D3 & Me gustan los desafíos y nuevas oportunidades. \\
\hline D4 & Prefiero realizar tareas que domino a la perfección y en las que me siento seguro. \\
\hline D5 & Me aventuro a hacer cosas nuevas y diferentes de lo que he hecho en el pasado. \\
\hline
\end{tabular}

Tabla 2-2 Declaraciones que evalúan la Persistencia

\begin{tabular}{|c|l|}
\hline D6 & $\begin{array}{l}\text { Cuando me enfrento a un problema difícil, dedico gran cantidad de tiempo en } \\
\text { encontrar una solución. }\end{array}$ \\
\hline D7 & Insisto hasta conseguir que otras personas hagan lo que yo quiero. \\
\hline D8 & $\begin{array}{l}\text { Cuando algo se interpone en lo que estoy tratando de hacer, persisto en mi } \\
\text { objetivo. }\end{array}$ \\
\hline D9 & Cuando me enfrento a serias dificultades, rápidamente cambio a otras actividades. \\
\hline D10 & $\begin{array}{l}\text { Trato diferentes formas para superar obstáculos que se interponen al logro de mis } \\
\text { metas. }\end{array}$ \\
\hline
\end{tabular}


Tabla 2-3 Declaraciones que evalúan el Cumplimiento de Compromisos

\begin{tabular}{|l|l|}
\hline D11 & Termino mi trabajo a tiempo. \\
\hline D12 & Cumplo con las promesas que hago. \\
\hline D13 & $\begin{array}{l}\text { Si es necesario, hago el trabajo de otras personas para cumplir y entregar un } \\
\text { trabajo a tiempo. }\end{array}$ \\
\hline D14 & $\begin{array}{l}\text { Cuando estoy haciendo un trabajo para otra persona me esfuerzo mucho por } \\
\text { lograr su satisfacción. }\end{array}$ \\
\hline D15 & $\begin{array}{l}\text { Mi familia y mi vida personal son más importantes que las fechas de entrega } \\
\text { de trabajos. }\end{array}$ \\
\hline
\end{tabular}

Tabla 2-4 Declaraciones que evalúan la Exigencia de Eficiencia y Calidad

\begin{tabular}{|l|l|}
\hline D16 & Me molesto cuando las cosas no se hacen correctamente. \\
\hline D17 & $\begin{array}{l}\text { Mi rendimiento en el trabajo es mejor que el de otras personas con las que } \\
\text { trabajo. }\end{array}$ \\
\hline D18 & Me molesta cuando pierdo el tiempo. \\
\hline D19 & $\begin{array}{l}\text { Me es difícil quedar totalmente satisfecho con la forma en que se hacen las } \\
\text { cosas. }\end{array}$ \\
\hline D20 & Me apuro para terminar labores, tanto en el trabajo como en el hogar. \\
\hline
\end{tabular}

Tabla 2-5 Declaraciones que evalúan el Asumir Riesgos

\begin{tabular}{|l|l|}
\hline D21 & Prefiero situaciones en las que puedo controlar al máximo el resultado final. \\
\hline D22 & Me involucro en algo nuevo solo si estoy seguro de que tendrá éxito. \\
\hline D23 & Antes de actuar considero mis posibilidades de éxito o fracaso. \\
\hline D24 & Llevo a cabo tareas arriesgadas. \\
\hline D25 & Hago cosas que otras personas consideran arriesgadas. \\
\hline
\end{tabular}

Tabla 2-6 Declaraciones que evalúan la Fijación de metas

\begin{tabular}{|l|l|}
\hline D26 & Me gusta pensar sobre el futuro. \\
\hline D27 & Pienso que es una pérdida de tiempo preocuparme sobre qué haré con mi vida. \\
\hline D28 & $\begin{array}{l}\text { Pienso que mientras más concreto es lo que quiero lograr mayores son mis } \\
\text { posibilidades de éxito. }\end{array}$ \\
\hline D29 & Cuento con un plan definido respecto a mi vida. \\
\hline D30 & Me preocupa tanto alcanzar mis metas semanales como mis metas anuales. \\
\hline
\end{tabular}


Tabla 2-7 Declaraciones que evalúan la Búsqueda de Información

\begin{tabular}{|l|l|}
\hline D31 & $\begin{array}{l}\text { Cuando comienzo una tarea o un proyecto nuevo, busco previamente toda la } \\
\text { información posible. }\end{array}$ \\
\hline D32 & $\begin{array}{l}\text { Busco el consejo de personas especialistas en las actividades en que yo me } \\
\text { estoy desempeñando. }\end{array}$ \\
\hline D33 & Actúo sin perder tiempo buscando información. \\
\hline D34 & $\begin{array}{l}\text { Cuando realizo un trabajo para alguien, hago preguntas para asegurarme que } \\
\text { entiendo lo que quiere. }\end{array}$ \\
\hline D35 & $\begin{array}{l}\text { Me valgo de varias fuentes de información al buscar ayuda para llevar a cabo } \\
\text { tareas o proyectos. }\end{array}$ \\
\hline
\end{tabular}

Tabla 2-8 Declaraciones que evalúan la Planificación sistemática y seguimiento

\begin{tabular}{|l|l|}
\hline D36 & Planifico un proyecto grande dividiéndolo en pequeñas tareas. \\
\hline D37 & $\begin{array}{l}\text { Para realizar un trabajo considero diferentes alternativas, con sus ventajas y } \\
\text { desventajas. }\end{array}$ \\
\hline D38 & $\begin{array}{l}\text { Trato de tomar en cuenta todos los problemas que pueden presentarse y } \\
\text { anticipo que hacer. }\end{array}$ \\
\hline D39 & $\begin{array}{l}\text { Me enfrento a problemas a medida que surgen, en vez de perder tiempo } \\
\text { tratando de anticiparlos. }\end{array}$ \\
\hline D40 & Si no resulta un determinado camino para enfrentar un problema, busco otro. \\
\hline
\end{tabular}

Tabla 2-9 Declaraciones que evalúan la Persuasión y construcción de redes de apoyo

\begin{tabular}{|l|l|}
\hline D41 & Logro que otros apoyen mis recomendaciones. \\
\hline D42 & Pienso poco en como influenciar a otras personas. \\
\hline D43 & Recurro a personas influyentes para alcanzar mis metas. \\
\hline D44 & $\begin{array}{l}\text { Para enfrentar problemas en el logro de mis metas, busco soluciones que } \\
\text { beneficien a todos los involucrados. }\end{array}$ \\
\hline D45 & $\begin{array}{l}\text { Puedo lograr que personas con firmes convicciones cambien de modo de } \\
\text { pensar. }\end{array}$ \\
\hline
\end{tabular}

\section{Tabla 2-10 Declaraciones que evalúan la Autoconfianza e Independencia}

\begin{tabular}{|l|l|}
\hline D46 & Confío que puedo tener éxito en cualquier actividad que me propongo realizar. \\
\hline D47 & $\begin{array}{l}\text { Cambio de manera de pensar si otras personas difieren enérgicamente con mis } \\
\text { puntos de vista. }\end{array}$ \\
\hline D48 & $\begin{array}{l}\text { Cuando estoy desempeñándome en algo difícil, me siento confiado en mi } \\
\text { triunfo. }\end{array}$ \\
\hline D49 & El trabajo que realizo es excelente. \\
\hline D50 & $\begin{array}{l}\text { Me mantengo firme en mis decisiones, aun cuando otras personas me } \\
\text { contradigan enérgicamente. }\end{array}$ \\
\hline
\end{tabular}


Tabla 2-11 Declaraciones para calcular el Factor de Corrección

\begin{tabular}{|l|l|}
\hline D51 & No importa con quién planifique, siempre lo escucho con atención. \\
\hline D52 & Me resiento cuando no logro lo que quiero. \\
\hline D53 & He sufrido fracasos en el pasado. \\
\hline D54 & En ciertas ocasiones, he sacado ventaja de otras personas. \\
\hline D55 & Cuando no sé algo, no tengo problemas en aceptarlo. \\
\hline
\end{tabular}

La puntuación para cada característica se obtiene de acuerdo con los cálculos de la Tabla 2-13.

Tabla 2-12 Cálculo de las puntuaciones preliminares

\begin{tabular}{|l|l|}
\hline \multicolumn{1}{|c|}{ Característica Emprendedora Personal } & \multicolumn{1}{c|}{ Fórmula } \\
\hline Búsqueda de Oportunidades e Iniciativa & D1 + D2 + D3 - D4 + D5 + 6 \\
\hline Persistencia & D6 + D7 + D8- D9 + D10 + 6 \\
\hline Cumplimiento de Compromisos & D11 + D12 + D13 + D14 - D15 + 6 \\
\hline Exigencia de Eficiencia y Calidad & D16 + D17 + D18 + D19 - D20 + 6 \\
\hline Asumir Riesgos & D21 - D22 + D23 + D24 + D25 + 6 \\
\hline Fijación de Metas & D26 - D27 + D28 + D29 + D30 + 6 \\
\hline Búsqueda de Información & D31 + D32 - D33 + D34 + D35 + 6 \\
\hline Planificación Sistemática y Seguimiento & D36 + D37 + D38 - D39 + D40 + 6 \\
\hline $\begin{array}{l}\text { Persuasión y Construcción de Redes de } \\
\text { Apoyo }\end{array}$ & D41 - D42 + D43 + D44 + D45 + 6 \\
\hline Autoconfianza e Independencia & D46 - D47 + D48 + D49 + D50 + 6 \\
\hline Factor de Corrección & D51- D52 - D53- D54 + D55 + 6 \\
\hline
\end{tabular}

De acuerdo con el puntaje obtenido en el factor de corrección se realizan ajustes a los puntajes preliminares de cada característica siguiendo las indicaciones de la Tabla 2-14.

Tabla 2-13 Ajuste a realizar de acuerdo con el Factor de Corrección

\begin{tabular}{|l|l|}
\hline Rango Obtenido & \multicolumn{1}{c|}{ Ajuste } \\
\hline $\mathbf{2 4 - 2 5}$ & restar 7 a cada característica \\
\hline $\mathbf{2 2 - 2 3}$ & restar 5 a cada característica \\
\hline $\mathbf{2 0 - 2 1}$ & restar 3 a cada característica \\
\hline $\mathbf{0 - 1 9}$ & restar 0 a cada característica \\
\hline
\end{tabular}


Para obtener el puntaje correspondiente a cada característica es valorada de manera individual de acuerdo con la Tabla 2-15 de modo a obtener el perfil emprendedor.

Tabla 2-14 Cálculo de las Puntuaciones

\begin{tabular}{|l|l|l|l|}
\hline \multicolumn{1}{|c|}{ Característica Emprendedora } & \multicolumn{1}{c|}{$\begin{array}{c}\text { Puntaje } \\
\text { Preliminar }\end{array}$} & Ajuste & $\begin{array}{c}\text { Total } \\
\text { Corregido }\end{array}$ \\
\hline $\begin{array}{l}\text { Búsqueda de Oportunidades e } \\
\text { Iniciativa }\end{array}$ & & \\
\hline Persistencia & & \\
\hline Cumplimiento de Compromisos & & \\
\hline Exigencia de Eficiencia y Calidad & & \\
\hline Asumir Riesgos & & \\
\hline Fijación de metas & & \\
\hline Búsqueda de Información & & \\
\hline $\begin{array}{l}\text { Planificación sistemática y } \\
\text { seguimiento }\end{array}$ & & \\
\hline $\begin{array}{l}\text { Persuasión y construcción de redes } \\
\text { de apoyo }\end{array}$ & & \\
\hline $\begin{array}{l}\text { Autoconfianza e Independencia } \\
\text { Factor de Corrección }\end{array}$ & & \\
\hline
\end{tabular}

\subsubsection{Desarrollo de las clases prácticas de laboratorio.}

Las actividades en común del desarrollo de ambos laboratorios son:

- Evaluación Previa a la Práctica

- Explicativa de la Práctica para desarrollar

- Realización de la Práctica

- Elaboración del Informe de la Práctica

- Evaluación Escrita

- Evaluación Oral

- Evaluación Práctica

- Presentación de un Trabajo Práctico Especial

Para lograr el fortalecimiento de las características emprendedoras personales, la propuesta para el laboratorio de QAI fue la modificación del desarrollo de dos clases prácticas en el laboratorio, quedando la distribución de las cuatro horas establecidas sufrió cambios como describe la Tabla 2.16. 
Tabla 2-15 Comparativo de la Planificación Anterior con la Propuest

\begin{tabular}{|l|c|c|}
\hline \multicolumn{1}{|c|}{ Actividad } & Planificación Anterior & Propuesta \\
\hline Prueba Pre Laboratorio & $20^{\prime}$ & $20^{\prime}$ \\
\hline Explicativa del Día & $20^{\prime}$ & $20^{\prime}$ \\
\hline Repartición de las Muestras & $5^{\prime}$ & 5 \\
\hline Análisis Muestra Individual & $\begin{array}{c}2^{\circ} 55^{\prime} \text { (dos o más } \\
\text { muestras) }\end{array}$ & $\begin{array}{c}1^{\circ} 30^{\prime} \text { (hasta dos } \\
\text { muestras) }\end{array}$ \\
\hline Análisis Muestra Grupal & --- & $1^{\circ} 30^{\prime}$ (una muestra) \\
\hline Elaboración del Informe & $10^{\prime}$ & 5 \\
\hline Cierre & $10^{\prime}$ & $10^{\prime}$ \\
\hline
\end{tabular}

El objetivo principal de cada práctica del laboratorio de QAI es la identificación de los iones presentes en una muestra. La modificación en la planificación se realizó en dos prácticas en la que los estudiantes debían de identificar de manera grupal una muestra que poseía una dificultad superior a la de las muestras individuales. Los casos analizados fueron de muestras inestables y muestras insolubles. La conformación de los grupos fue por mesada de seis alumnos. Para el logro de los objetivos, siguieron las fases del ABP descrito en la Tabla 2-17.

Tabla 2-16 Fases del ABP en las Muestras analizadas

\begin{tabular}{|c|c|c|}
\hline FASES DEL ABP & MUESTRA 1 & MUESTRA 2 \\
\hline $\begin{array}{l}\text { Aclarar conceptos y } \\
\text { términos }\end{array}$ & $\begin{array}{l}\text { La muestra corresponde a un } \\
\text { catión del grupo I. }\end{array}$ & $\begin{array}{l}\text { La muestra corresponde a } \\
\text { un catión del grupo IV. }\end{array}$ \\
\hline Definir el problema & $\begin{array}{l}\text { No se obtienen los resultados } \\
\text { de acuerdo con lo previsto } \\
\text { por la bibliografía. }\end{array}$ & $\begin{array}{l}\text { La muestra sólida no se } \\
\text { disuelve en agua destilada. }\end{array}$ \\
\hline Analizar el problema & $\begin{array}{l}\text { Los iones probables son el } \\
\text { Plomo }(+2) \text {, Plata }(+1) \text { y } \\
\text { Mercurio }(+1) \text {. }\end{array}$ & $\begin{array}{l}\text { Los iones probables son el } \\
\text { Calcio }(+2) \text {, Estroncio }(+2) \\
\text { y Bario }(+2) \text {. }\end{array}$ \\
\hline $\begin{array}{l}\text { Realizar un resumen } \\
\text { sistemático con varias } \\
\text { explicaciones al análisis } \\
\text { del paso anterior }\end{array}$ & $\begin{array}{l}\text { Los cationes plata y plomo } \\
\text { en solución son bastante } \\
\text { estables. El catión } \\
\text { mercurioso se encuentra } \\
\text { ionizado solamente en } \\
\text { soluciones ácidas, ya que, al } \\
\text { aumentar el pH se produce } \\
\text { un precipitado negro de } \\
\text { óxido mercurioso, que } \\
\text { dismuta espontáneamente a } \\
\text { óxido mercúrico y mercurio } \\
\text { elemental. }\end{array}$ & $\begin{array}{l}\text { Para disolver la sustancia } \\
\text { sólida, se examina la } \\
\text { solubilidad en: agua } \\
\text { destilada fría, agua } \\
\text { destilada caliente, ácido } \\
\text { clorhídrico diluido, ácido } \\
\text { clorhídrico concentrado, } \\
\text { ácido nítrico diluido, ácido } \\
\text { nítrico concentrado y en } \\
\text { agua regia. }\end{array}$ \\
\hline
\end{tabular}




\begin{tabular}{|l|l|l|}
\hline $\begin{array}{l}\text { Formular los resultados } \\
\text { del aprendizaje } \\
\text { esperados (objetivos) }\end{array}$ & $\begin{array}{l}\text { Tratamiento adecuado de } \\
\text { una muestra que contiene } \\
\text { iones de Mercurio (+1). }\end{array}$ & $\begin{array}{l}\text { Elección del solvente } \\
\text { adecuado para el sólido. }\end{array}$ \\
\hline $\begin{array}{l}\text { Buscar información } \\
\text { adicional fuera del } \\
\text { grupo o estudio } \\
\text { individual }\end{array}$ & $\begin{array}{l}\text { Consulta sobre el } \\
\text { comportamiento de los iones } \\
\text { en disolución en otras } \\
\text { bibliografías o documentos } \\
\text { disponibles en la web. }\end{array}$ & $\begin{array}{l}\text { Consulta sobre valores de } \\
\text { productos de solubilidad y } \\
\text { la influencia de ácidos } \\
\text { fuertes y débiles sobre la } \\
\text { solubilidad (o el pH). }\end{array}$ \\
\hline $\begin{array}{l}\text { Síntesis de la } \\
\text { información recogida y } \\
\text { elaboración del informe } \\
\text { sobre los conocimientos } \\
\text { adquiridos }\end{array}$ & $\begin{array}{l}\text { El análisis se puede realizar } \\
\text { sobre la muestra sólida para } \\
\text { evitar que se oxide } \\
\text { rápidamente en solución } \\
\text { acuosa o preparar la solución } \\
\text { con ácido nítrico diluido. }\end{array}$ & $\begin{array}{l}\text { El ácido clorhídrico diluido. } \\
\text { adido con }\end{array}$ \\
\hline
\end{tabular}

Para el laboratorio de QAII, la modificación en la planificación consistió en la presentación de un trabajo práctico especial por mesada (ocho alumnos) en la que debían elaborar un video tutorial de la cuantificación de un analito en una muestra definiendo la técnica específica a ser utilizada. El mismo debía de ser publicado en la red social youtube con el contenido descrito en la Tabla 2.18. Además, entregaron un informe final en formato pdf con el esquema de la Tabla 2.19. En el anexo de este especificaron los roles que desempeñó cada uno.

\section{Tabla 2-17 Contenido del Video Tutorial}

Presentación de la Volumetría

Preparación de las Soluciones (cálculos, cuidados y almacenamiento)

Montaje de los equipos para la valoración

Valoración

Demostración de los cálculos

\section{Tabla 2-18 Contenido del Informe Escrito}

Portada

Título del Trabajo

Objetivo General

Objetivos Específicos

Fundamentos Teóricos

Materiales y Reactivos

Procedimiento

Tabla de Datos

Cálculos

Discusión de Resultados

Conclusión

Bibliografía

Anexos (tabla con la distribución de tareas, imágenes, enlace del video tutorial) 
En la Tabla 2-20 se encuentran los temas distribuidos con los enlaces de los videos tutoriales disponibles en la red social YOUTUBE y el de los informes escritos en formato pdf en la plataforma de SLIDESHARE.

Tabla 2-19 Listado de Temas con los enlaces de los videotutoriales y los informes

\begin{tabular}{|c|c|c|c|}
\hline Muestra & Técnica & Video Tutorial & Informe Escrito \\
\hline $\begin{array}{l}\text { Hidróxido } \\
\text { de Sodio }\end{array}$ & $\begin{array}{l}\text { Volumetría de } \\
\text { Neutralización - } \\
\text { Método Directo }\end{array}$ & $\begin{array}{l}\text { https://www.youtu } \\
\underline{\text { be.com/watch?v=f }} \\
\underline{\text { XSV-p4K2DM }}\end{array}$ & $\begin{array}{l}\text { https://es.slideshare.net/Noel } \\
\text { iaCenturion/volumetra-de- } \\
\text { neutralizacin-mtodo-directo- } \\
\text { para-la-valoracin-de-na-oh }\end{array}$ \\
\hline $\begin{array}{l}\text { Ácido } \\
\text { Sulfúrico }\end{array}$ & $\begin{array}{l}\text { Volumetría de } \\
\text { Neutralización - } \\
\text { Método Directo } \\
\text { y Método por } \\
\text { Retroceso }\end{array}$ & $\begin{array}{l}\text { https://www.youtu } \\
\text { be.com/watch?v=L } \\
\text { rFE- } \\
\text { yvJbbU\&feature =y } \\
\text { outu.be }\end{array}$ & $\begin{array}{l}\text { https://es.slideshare.net/Noel } \\
\text { iaCenturion/volumetra-de- } \\
\text { neutralizacin-mtodo-directo-- } \\
\text { y-por-retroceso-del-cido- } \\
\text { sulfrico }\end{array}$ \\
\hline Bicarbonato & $\begin{array}{l}\text { Volumetría de } \\
\text { Neutralización - } \\
\text { Mezcla de } \\
\text { Álcalis }\end{array}$ & $\begin{array}{l}\frac{\text { https://www.youtu }}{\text { be.com/watch?v=G }} \\
\text { ZijhSji30Q\&featur } \\
\text { e=youtu.be }\end{array}$ & $\begin{array}{l}\text { https://es.slideshare.net/Noel } \\
\text { iaCenturion/volumetra-de- } \\
\text { neutralizacin-mezcla-de- } \\
\text { lcalis-bicarbonato }\end{array}$ \\
\hline Hidróxido & $\begin{array}{l}\text { Volumetría de } \\
\text { Neutralización - } \\
\text { Mezcla de } \\
\text { Álcalis }\end{array}$ & $\begin{array}{l}\frac{\text { https://www.youtu }}{\text { be.com/watch?v=C }} \\
\underline{\text { bg69pRad9Q\&feat }} \\
\underline{\text { ure=youtu.be }}\end{array}$ & $\begin{array}{l}\text { https://es.slideshare.net/Noel } \\
\text { iaCenturion/volumetra-de- } \\
\text { neutralizacin-mezcla-de- } \\
\underline{\text { lcalis-hidrxido }}\end{array}$ \\
\hline Carbonato & $\begin{array}{l}\text { Volumetría de } \\
\text { Neutralización - } \\
\text { Mezcla de } \\
\text { Álcalis }\end{array}$ & $\begin{array}{l}\text { https://www.youtu } \\
\text { be.com/watch?v=h } \\
\text { 8P- } \\
\text { V25hwQg\&feature } \\
\text { =youtu.be }\end{array}$ & $\begin{array}{l}\text { https://es.slideshare.net/Noel } \\
\text { iaCenturion/volumetra-de- } \\
\text { neutralizacin-mezcla-de- } \\
\underline{\text { lcalis-carbonato }}\end{array}$ \\
\hline Oxalato & $\begin{array}{l}\text { Volumetría } \\
\text { REDOX - } \\
\text { Permanganimetrí } \\
\text { a }\end{array}$ & $\begin{array}{l}\underline{\text { https://www.youtu }} \\
\text { be.com/watch?v=fo } \\
\text { BgsoWel9Y }\end{array}$ & $\begin{array}{l}\text { https://es.slideshare.net/Noel } \\
\text { iaCenturion/volumetra- } \\
\text { redox-permanganimetria }\end{array}$ \\
\hline $\begin{array}{l}\text { Ácido } \\
\text { Acético }\end{array}$ & $\begin{array}{l}\text { Titulación } \\
\text { Potenciometrica }\end{array}$ & $\begin{array}{l}\frac{\text { https://www.youtu }}{\text { be.com/watch?v=v }} \\
\underline{\text { h2PYlnk9QA }}\end{array}$ & $\begin{array}{l}\text { https://es.slideshare.net/Noel } \\
\text { iaCenturion/volumetra-de- } \\
\text { neutralizacin-potenciometra- } \\
\text { cido-dbil }\end{array}$ \\
\hline $\begin{array}{l}\text { Nitrato de } \\
\text { Cobalto }\end{array}$ & $\begin{array}{l}\text { Espectrofotomet } \\
\text { ría (utilización } \\
\text { de los } \\
\text { espectrofotómetr } \\
\text { os) }\end{array}$ & $\begin{array}{l}\frac{\text { https://www.youtu }}{\text { be.com/watch?v=6 }} \\
\underline{\text { U3KYIm0178 }}\end{array}$ & $\begin{array}{l}\text { https://es.slideshare.net/Noel } \\
\text { iaCenturion/espectrofotomet } \\
\text { ra-83400870 }\end{array}$ \\
\hline
\end{tabular}


En la Tabla 2.21 se detalla cómo influyen las metodologías en el fortalecimiento de cada característica emprendedora personal.

Tabla 2-20 Influencia de las Metodologías aplicadas en el Laboratorio en las CEP's

\begin{tabular}{|l|l|}
\hline $\begin{array}{c}\text { Característica } \\
\text { Emprendedora } \\
\text { Personal }\end{array}$ & \multicolumn{1}{|c|}{ Influencia del ABP y AC } \\
\hline $\begin{array}{l}\text { Búsqueda de } \\
\text { Oportunidades e } \\
\text { Iniciativa }\end{array}$ & $\begin{array}{l}\text { Los estudiantes deben analizar el problema para definir las } \\
\text { acciones a seguir. A través de la consulta a diferentes bibliografías } \\
\text { se obtienen diferentes posibles soluciones. }\end{array}$ \\
\hline Persistencia & $\begin{array}{l}\text { Si los resultados preliminares de la práctica poseen desviaciones } \\
\text { significativas, deben de repetir nuevamente hasta lograr el } \\
\text { objetivo de la práctica. Durante las filmaciones, las escenas debían } \\
\text { de repetirse hasta lograr la toma adecuada para el video. }\end{array}$ \\
\hline $\begin{array}{l}\text { Cumplimiento de } \\
\text { Compromisos }\end{array}$ & $\begin{array}{l}\text { Se definen plazos para la finalización de las prácticas, la } \\
\text { presentación de los informes y tutoriales. Asimismo, deben de } \\
\text { respetar la distribución de las tareas asegurando que cada uno } \\
\text { contribuya su parte. }\end{array}$ \\
\hline $\begin{array}{l}\text { Exigencia de } \\
\text { Eficiencia y } \\
\text { Calidad }\end{array}$ & $\begin{array}{l}\text { El informe final escrito y el video tutorial poseían indicadores pre- } \\
\text { establecidos para su elaboración y se hicieron revisiones antes de } \\
\text { ser entregados finalmente. }\end{array}$ \\
\hline $\begin{array}{l}\text { Asumir Riesgos } \\
\text { Fijación de metas }\end{array}$ & $\begin{array}{l}\text { Para la identificación de los analitos debían de realizar diferentes } \\
\text { pruebas. } \\
\text { Una vez recibida la muestra, los objetivos deben ser definidos de } \\
\text { modo a enfocar el trabajo. }\end{array}$ \\
\hline $\begin{array}{l}\text { Búsqueda de } \\
\text { Información }\end{array}$ & $\begin{array}{l}\text { Los estudiantes debían de consultar diferentes bibliografías para } \\
\text { complementar la información del manual del laboratorio. }\end{array}$ \\
\hline $\begin{array}{l}\text { Planificación } \\
\text { sistemática y } \\
\text { seguimiento }\end{array}$ & $\begin{array}{l}\text { Los procedimientos, los roles y las actividades debían de ser } \\
\text { planificadas de manera ordenada asegurando que cada estudiante } \\
\text { colabore. }\end{array}$ \\
\hline $\begin{array}{l}\text { Persuasión y } \\
\text { construcción de } \\
\text { redes de apoyo }\end{array}$ & $\begin{array}{l}\text { Ambas metodologías utilizan el trabajo en equipo, por lo que las } \\
\text { actitudes y habilidades sociales influyeron en cada fase del trabajo. }\end{array}$ \\
\hline $\begin{array}{l}\text { Independencia } \\
\text { ana vez distribuida la tarea, cada integrante debía ser capaz de } \\
\text { contribución era trascendental en el logro del objetivo. }\end{array}$ \\
\hline andianza e
\end{tabular}

\subsubsection{Medición Final de las Características Emprendedoras Personales.}

A fin de del segundo semestre del 2017, cuando el laboratorio de la cátedra de Química Analítica II concluyó, se les entregó nuevamente el cuestionario con las 55 declaraciones breves para que los estudiantes puedan volver a evaluarse.

\subsubsection{Análisis de los resultados}

Al final del Cuestionario CEP's se elaboró una Gráfica Radial por estudiante, similar a la Figura 2-1, en la que se situó las puntuaciones obtenidas tanto al inicio como al fin de 
modo a visualizar el cambio, de acuerdo con los resultados se pudo valorar a cada estudiante considerando la Tabla 2.22.

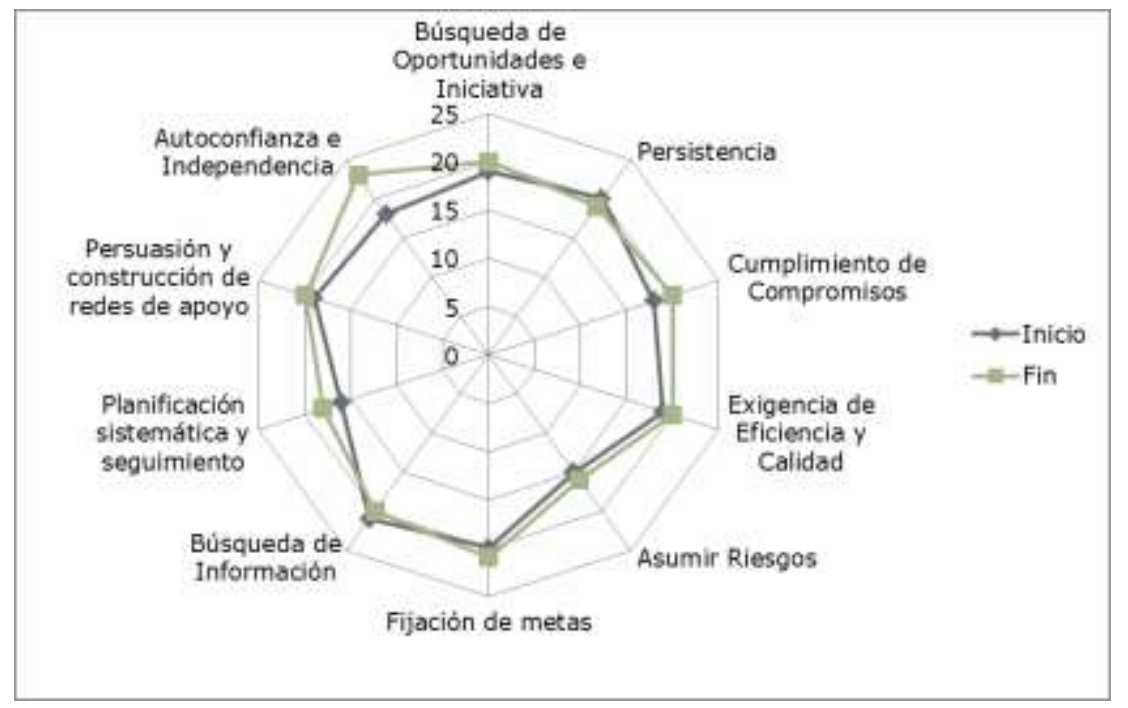

Figura 2-1 Comparativo de las Puntuaciones de las CEP's Iniciales y Finales de un estudiante.

Es importante destacar que ninguna persona posee nivel superior en todas las categorías y que a la hora de plantear un emprendimiento es recomendable asociarse con las personas que tengan fortalezas donde uno tiene debilidades (Briasco, 2014).

Tabla 2-21 Valoración de los resultados de las puntuaciones

\begin{tabular}{|l|l|}
\hline Resultados & \multicolumn{1}{c|}{ Explicación } \\
Menores e igual a 10 & $\begin{array}{l}\text { Se considera esta característica como debilidad o carencia, } \\
\text { esto significa que tiene que trabajar para mejorarla. Se debe } \\
\text { revisar las preguntas de la característica y evaluar qué se } \\
\text { puede hacer para obtener un mejor puntaje. }\end{array}$ \\
\hline Entre 11 y 15 & $\begin{array}{l}\text { Tiene un nivel medio en esa característica. No obstante, se } \\
\text { debe tratar de mejorar estas características hasta obtener un } \\
\text { nivel superior. }\end{array}$ \\
\hline Mayores a 16 & $\begin{array}{l}\text { Se posee un nivel superior en esa categoría. Cuanto más } \\
\text { cercano el puntaje al 25 se encuentra más desarrollada esta } \\
\text { característica. }\end{array}$ \\
\hline
\end{tabular}

Para la discusión de la influencia de las metodologías aplicadas sobre las CEP's, se realizó un análisis estadístico descriptivo de las medidas de tendencia central y un análisis paramétrico para determinar si existió una diferencia significativa. 


\section{RESULTADOS Y DISCUSIÓN}

\subsection{Análisis Individual de las CEP's en el Inicio y el Fin}

Una vez que el cuestionario de autoevaluación fue completado por cada estudiante al inicio del semestre que se cursó QAI y al fin del semestre correspondiente a QAII, los resultados de cada CEP's se pudieron evaluar de manera individual para verificar su variación en el transcurso del año. A continuación, se discute el comparativo del perfil emprendedor de 3 casos.

\subsubsection{Comparativo Estudiante 1}

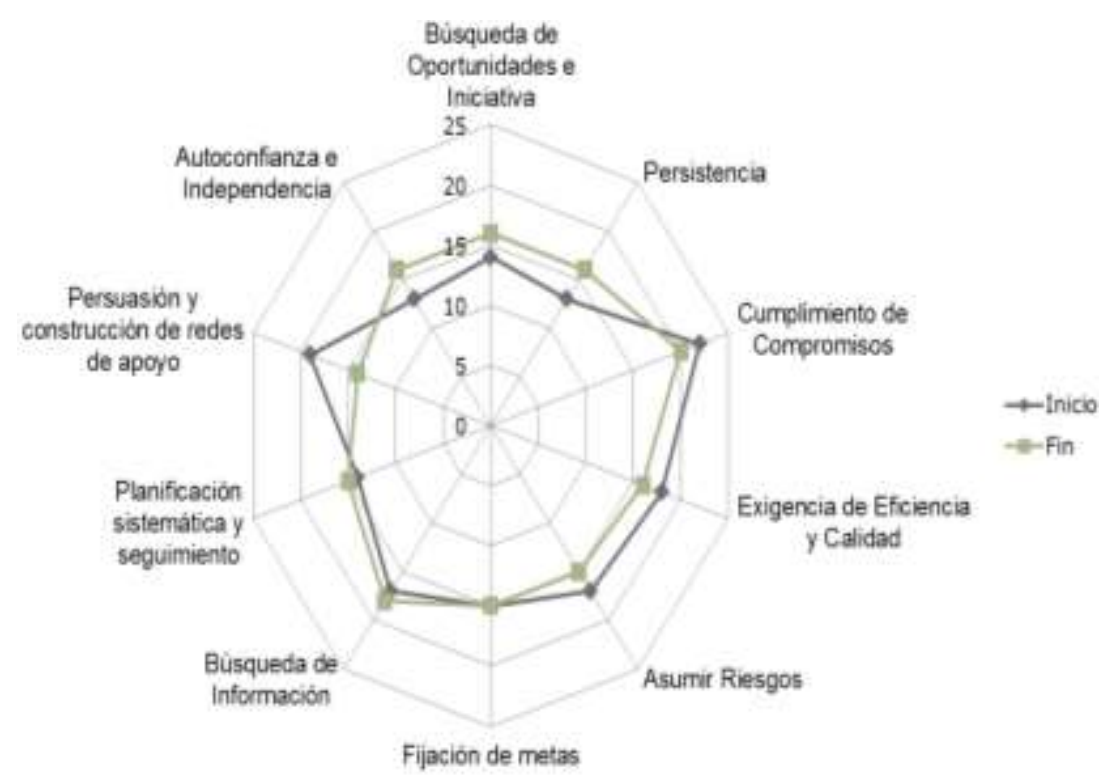

Figura 3-2 Comparativo de las CEP's del Inicio y Fin para el Estudiante 1

En el inicio, las características de la Búsqueda de Oportunidades e Iniciativa, Persistencia, Planificación Sistemática y Seguimiento y Autoconfianza e Independencia, presentan un nivel medio, menor a 16 puntos, por lo que debían de ser fortalecidas para alcanzar el nivel alto, situación que ocurrió comparando con las mediciones en el fin. Además, se pudo observar que las características de Cumplimiento de Compromisos, Exigencia de Eficiencia y Calidad, Asumir Riesgos, Persuasión y Construcción de Redes de Apoyo registraron una disminución, esto pudo deberse a la percepción más optimista que el estudiante poseía a inicio del semestre, mientras que al finalizar el año, influenciado por las dificultades atravesadas, se puntuó con valores menores. 


\subsubsection{Comparativo Estudiante 10.}

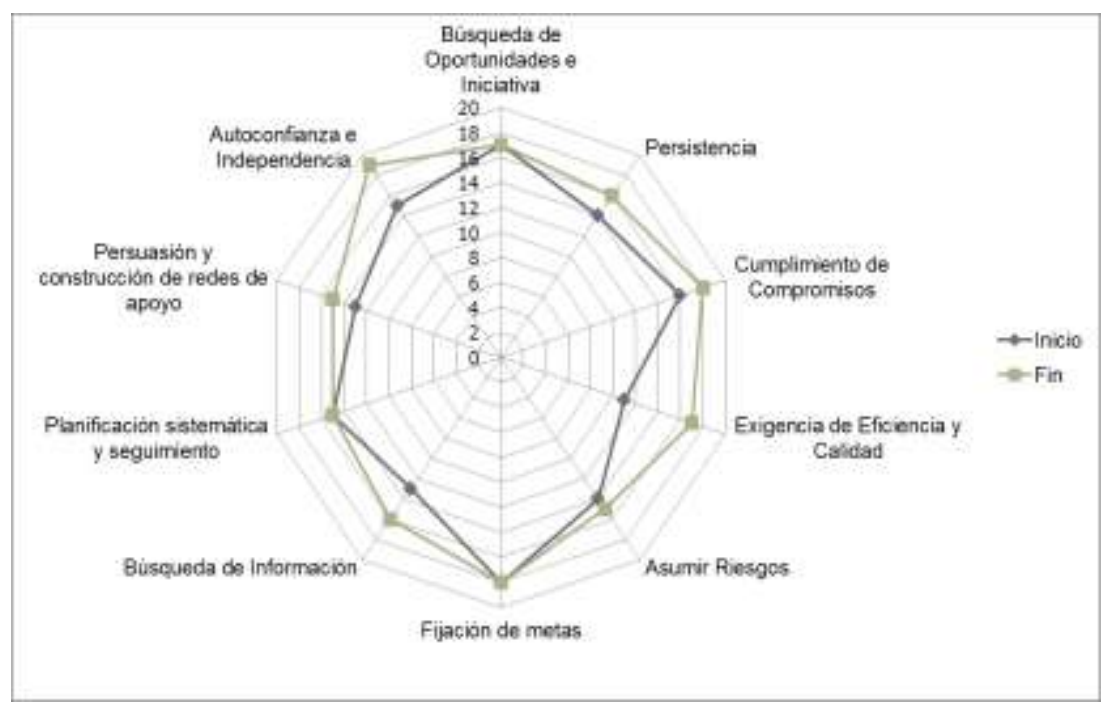

Figura 3-3 Comparativo de las CEP's en el Inicio y en el Fin del Estudiante 10.

En el Inicio, las características que registran un nivel medio son las de Persistencia, Asumir Riesgos, Exigencia de Eficiencia y Calidad, Búsqueda de Información, Planificación Sistemática y Seguimiento, Persuasión y Construcción de Redes de Apoyo y Autoconfianza e Independencia. Búsqueda de Oportunidades e Iniciativa, Cumplimiento de Compromisos y Fijación de Metas ya se encontraban en el nivel alto. Comparando con los puntajes finales, se verifica que se mantuvieron en el nivel medio la Planificación Sistemática y Seguimiento (se mantuvo con la misma puntuación) y Asumir Riesgos, Persuasión y Construcción de Redes de Apoyo. La característica de Exigencia de Calidad y Eficiencia que, inicialmente obtuvo el menor puntaje, solo 11, aumentó a 17 por lo que se pudo observar un gran fortalecimiento. La característica de Autoconfianza e Independencia registró la mayor puntuación en el fin, la cual se ve influenciada fuertemente por la experiencia y los conocimientos adquiridos, tanto teóricos como prácticos, dotándole de mayor seguridad en su desempeño y realización de los trabajos. 


\subsubsection{Comparativo Estudiante 43.}

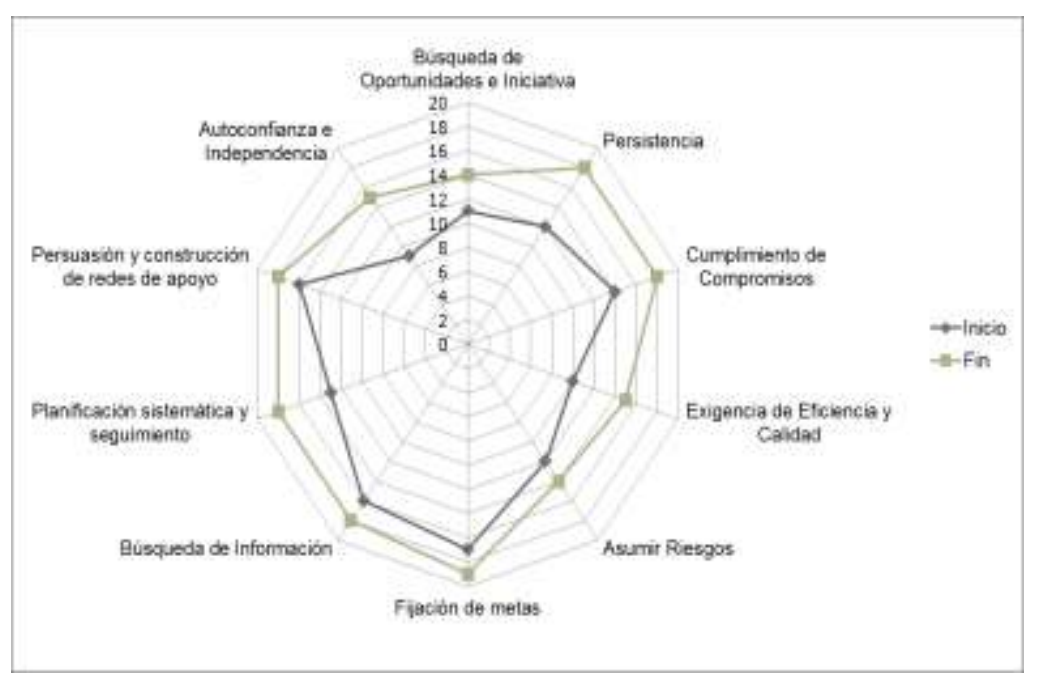

Figura 3-4 Comparativo de las CEP's en el Inicio y en el Fin del Estudiante 43.

En este caso, todas las características presentaron un fortalecimiento. En el Inicio, las características de Exigencia de Eficiencia y Calidad y Autoconfianza e Independencia se encontraban en el nivel bajo, las cuales registraron un crecimiento llegando al nivel medio. Las que obtuvieron el mayor aumento son la Persistencia y la Autoconfianza e Independencia, las cuales, a través de las vivencias y obstáculos superados, fueron afianzadas.

Este estudiante, en el primer semestre tuvo una participación más pasiva en el desarrollo de los laboratorios, tanto en el relacionamiento con los pares como con los docentes del laboratorio, mientras que, en el segundo semestre ya demostró una faceta más participativa, llegando inclusive a ser delegado del grupo y aportando interesantes alternativas para la resolución de problemas.

\subsection{Análisis Descriptivo de las Medidas de Tendencia Central}

Para el análisis descriptivo se realizó un comparativo de las observaciones al inicio y al fin de cada característica con los resultados de todos los estudiantes.

\subsubsection{Comparativo de Media}

En la Figura 3-5 se observa que las características de Fijación de Metas, Búsqueda de Información y Persuasión y construcción de redes de apoyo sufrieron una disminución en el promedio, esto puede deberse que, a inicio del año lectivo, cuando las actividades todavía no son muchas ni abrumadoras, el estudiante posee más tiempo para poder organizarse, tanto individual como grupalmente. 


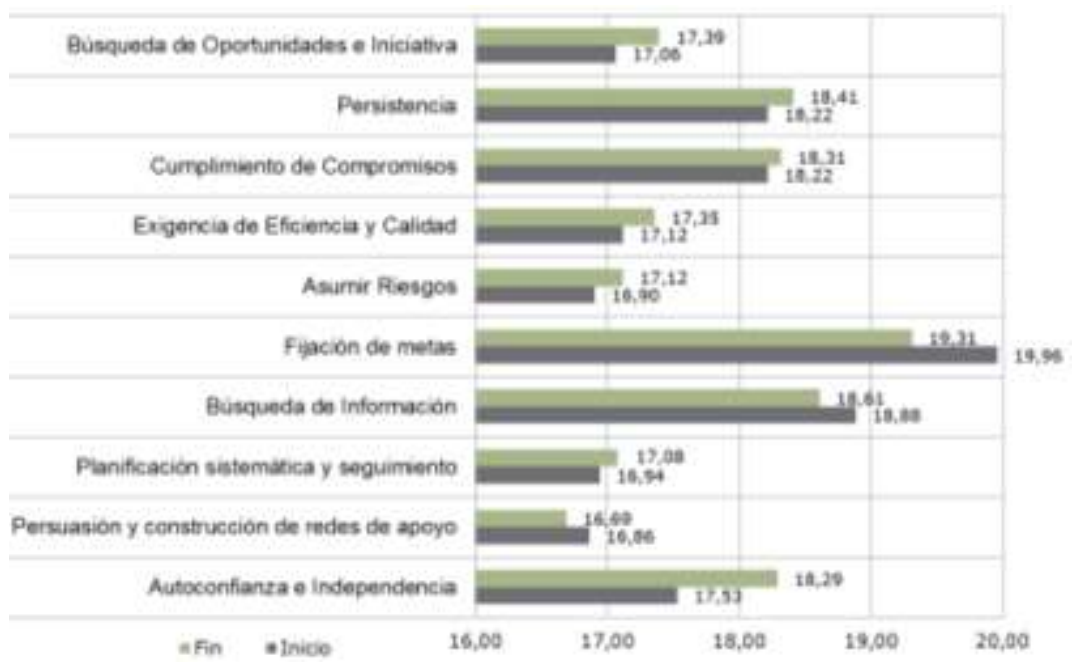

Figura 3-5 Comparativo de Media del Inicio y Fin

Las demás características presentaron un aumento de la media por lo que se pudo decir que fueron fortalecidas.

\subsubsection{Comparativo de Mediana}

La Figura 3-6 indica que las características de Búsqueda de Información y Persuasión y Construcción de redes de apoyo sufrieron una disminución en los valores que dividen la distribución por la mitad.

La característica que obtuvo un aumento en la mediana fue el de Autoconfianza e Independencia, esto puedo deberse a que el estudiante, a medida que va avanzando de nivel y afianza la experiencia práctica se siente más seguro y posee mayor cantidad de conocimientos y habilidades. Las demás variables se mantuvieron iguales en su medida central.

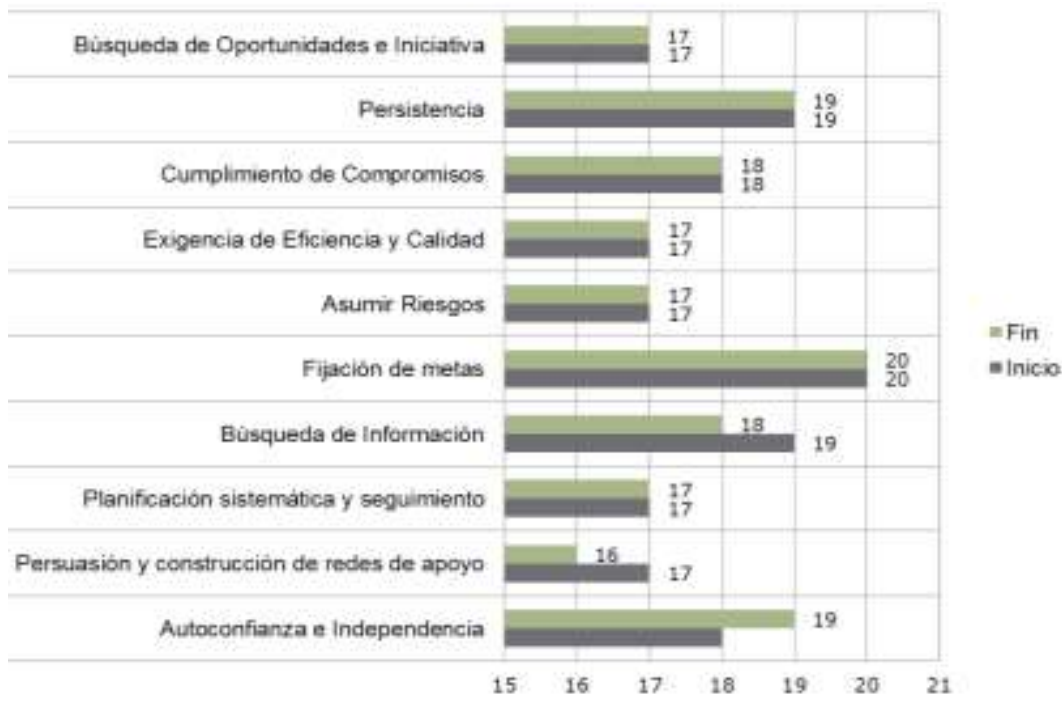

Figura 3-6 Comparativo de Mediana en el Inicio y en el Fin 


\subsubsection{Comparativo de Moda}

Según la Figura 3-7, las características de Fijación de Metas y Búsqueda de Información sufrieron una disminución en la puntuación con mayor frecuencia esto pudo deberse que a finales de año, el estudiante, en general, no se detiene a analizar los objetivos que desea alcanzar, tanto a corto, mediano o largo plazo, sino que, considera que el calendario de las actividades ya lo limitan, así también la percepción corta del tiempo influye en restringir la búsqueda de más información como para afrontar las situaciones.

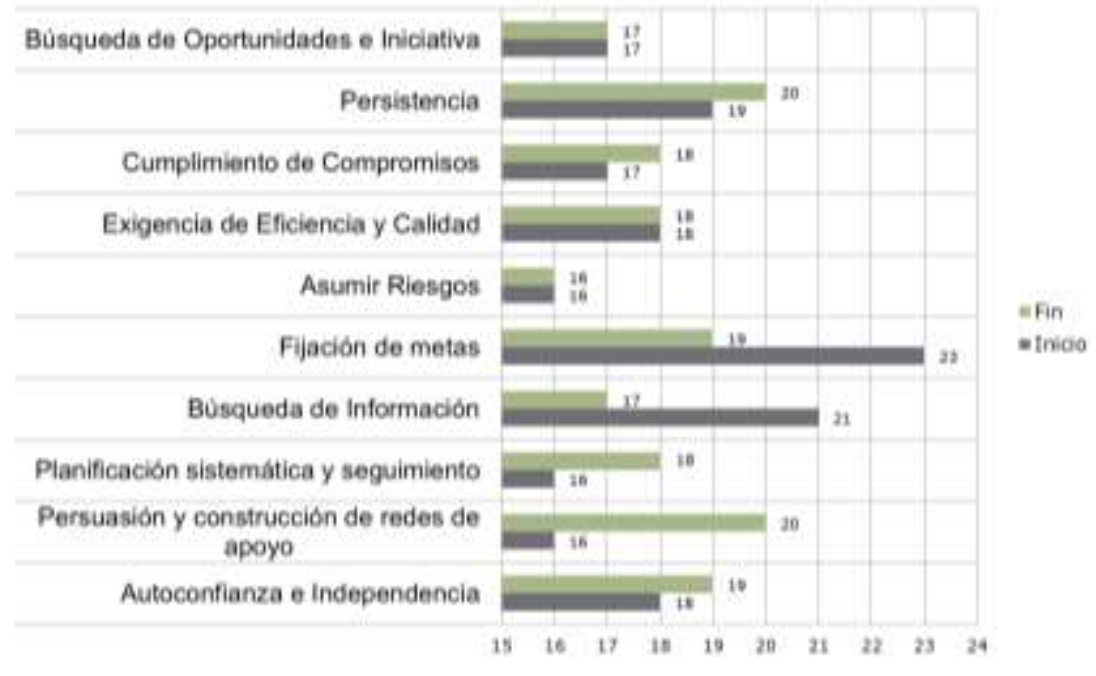

Figura 3-8 Comparativo de la Moda en el Inicio y en el Fin

No registraron variación de la moda la Búsqueda de Oportunidades e Iniciativa, Exigencia de Calidad y Eficiencia y el Asumir Riesgos.

Las demás características presentaron un aumento en el valor que se repite con mayor frecuencia al finalizar el segundo semestre.

\subsection{Pruebas de Normalidad}

La Prueba de Normalidad consiste en una prueba de hipótesis para examinar si las observaciones siguen o no una distribución normal.

$\boldsymbol{H}_{\boldsymbol{o}}$ (Hipótesis nula): Los datos siguen una distribución normal.

Ha (Hipótesis Alternativa): Los datos no siguen una distribución normal.

\subsubsection{Prueba Shapiro-Wilk}

Siendo la hipótesis nula que la población está distribuida normalmente, si el p-valor es menor a alfa (nivel de significancia) entonces la hipótesis nula es rechazada (se concluye que los datos no vienen de una distribución normal). Si el p-valor es mayor a alfa, no se rechaza la hipótesis y se concluye que los datos siguen una distribución normal. 
Tabla 3-22 p-valor de Shapiro Wilk de las CEP's en el Inicio y en el Fin

\begin{tabular}{|l|c|c|}
\hline \multicolumn{1}{|c|}{ Características Emprendedoras Personales } & $\begin{array}{c}\text { p-valor } \\
\text { Inicio }\end{array}$ & p-valor Fin \\
\hline Búsqueda de Oportunidades e Iniciativa & 0,13 & 0,10 \\
\hline Persistencia & 0,69 & 0,10 \\
\hline Cumplimiento de Compromisos & 0,48 & 0,15 \\
\hline Exigencia de Eficiencia y Calidad & 0,17 & 0,09 \\
\hline Asumir Riesgos & 0,06 & 0,07 \\
\hline Fijación de metas & 0,08 & 0,20 \\
\hline Búsqueda de Información & 0,06 & 0,37 \\
\hline Planificación sistemática y seguimiento & 0,43 & 0,06 \\
\hline Persuasión y construcción de redes de apoyo & 0,41 & 0,17 \\
\hline Autoconfianza e Independencia & 0,39 & 0,11 \\
\hline
\end{tabular}

La Tabla 3-23 indica que todas las mediciones de las características antes y después poseen el p-valor mayor a 0,05 por lo que se aceptó la hipótesis nula de que las observaciones seguían una distribución normal.

\subsection{Análisis Paramétrico}

Como los datos poseían una distribución normal, se efectuó un análisis paramétrico. A través de la prueba t se evaluó estadísticamente si las mediciones diferían de manera significativa.

\subsubsection{Pruebas t de Student de Muestras Relacionadas}

Se dispuso de los resultados de las características emprendedoras personales individuales al inicio y al final del año, por lo que se pudo realizar una prueba t para muestras relacionadas, comparando las medias de dos series de mediciones realizadas sobre las mismas unidades estadísticas.

$H_{o}$ (Hipótesis nula): El desarrollo de las clases prácticas de los laboratorios de Química Analítica I y Química Analítica II no fortalecen las características emprendedoras personales de los estudiantes. (No existe diferencia significativa entre las mediciones). 
$H_{a}$ (Hipótesis Alternativa): El desarrollo de las clases prácticas de los laboratorios de Química Analítica I y Química Analítica II fortalecen las características emprendedoras personales de los estudiantes. (Existe diferencia significativa entre las mediciones).

Debido a que lo que se deseaba comprobar fue el fortalecimiento, es decir, un incremento de los valores de las variables se trató de un contraste unilateral derecho.

La regla de decisión a tener en cuenta es que si el p-valor es menor al nivel de significación $\alpha(\alpha=0,05)$, se rechaza la hipótesis nula, y si el p valor es mayor al nivel de significación $\alpha$, se acepta la hipótesis nula, por lo que se concluye que no existe diferencia significativa con un nivel de confianza del $95 \%$.

Tabla 3-23 p-valor de la prueba t para muestras relacionadas

\begin{tabular}{|l|c|}
\hline Características & p valor \\
\hline Búsqueda de Oportunidades e Iniciativa & 0,16 \\
\hline Persistencia & 0,29 \\
\hline Cumplimiento de Compromisos & 0,40 \\
\hline Exigencia de Eficiencia y Calidad & 0,25 \\
\hline Asumir Riesgos & 0,29 \\
\hline Fijación de metas & 0,06 \\
\hline Búsqueda de Información & 0,22 \\
\hline Planificación sistemática y seguimiento & 0,38 \\
\hline Persuasión y construcción de redes de apoyo & 0,32 \\
\hline Autoconfianza e Independencia & 0,04 \\
\hline
\end{tabular}

Teniendo en cuenta la Tabla 3-24, sólo en Autoconfianza e Independencia se obtuvo un p-valor menor a 0,05 por lo que la hipótesis nula se rechazó, aceptando la hipótesis alternativa, comprobándose el fortalecimiento.

Para las demás características, el p-valor fue mayor que 0,05 por lo que la hipótesis nula se aceptó, no existiendo la diferencia significativa entre las mediciones.

\section{CONCLUSIÓN O CONSIDERACIONES FINALES}

Las dos metodologías propuestas promueven el aprendizaje centrado en el alumno basando el trabajo en pequeños grupos, por lo que tanto las fases del $\mathrm{ABP}$ como el esquema organizativo del AC influencian en cada una de las CEP's. La búsqueda de oportunidades e iniciativa se fortalecen cuando los estudiantes analizan el problema para 
definir los pasos a seguir para solucionarlo. Aplican la persistencia cuando los resultados preliminares de la práctica poseen desviaciones significativas o se incurrieron en errores por lo que deben de realizar nuevamente los análisis. Las fechas definidas para las revisiones y entrega de los informes así también como la distribución de las actividades para cada miembro del grupo influyen en el Cumplimiento de Compromisos. Los indicadores establecidos para la realización de cada trabajo ayudan a que la Exigencia de Calidad y Eficiencia tenga parámetros con el cual ser medidos. La investigación sobre posibles soluciones para la resolución del problema alienta al estudiante a Asumir Riesgos de manera calculada. La Fijación de Metas es importante para que el trabajo de cada integrante esté direccionado. La Búsqueda de Información es crucial para fundamentar la técnica a ser utilizada. La dificultad del problema obliga a que las se distribuyan con una Planificación Sistemática. Como los estudiantes poseen diferentes niveles de habilidades y conocimientos, cada miembro del equipo es responsable no solo de su aprendizaje, sino también de ayudar a sus compañeros a aprender, fortaleciendo así, la Persuasión y Construcción de Redes de Apoyo. Finalmente, el logro de los objetivos, los nuevos contenidos estudiados, las destrezas desarrolladas y con los resultados apreciables de la contribución de cada uno, afianzan la Autoconfianza e Independencia.

A través de la distribución del cuestionario de autoevaluación a los estudiantes para su llenado al inicio del Laboratorio de QAI y al finalizar el Laboratorio de QAII se pudo obtener el perfil emprendedor de cada alumno, definiendo si la CEP se encuentra en un nivel bajo, medio o alto. Además, se evaluó si las características fueron fortalecidas o no. El instrumento utilizado es susceptible a la percepción de uno mismo por el estado anímico del momento, ya que, cuando inicia el semestre, el alumno, de manera optimista, se propone a ser más organizado en su estudio, asistir y participar de todas las clases, entregar las tareas de manera excelente, utilizar de manera efectiva su tiempo, aportar en los trabajos en equipo, etc., pero, a medida que transcurre el período lectivo, podría no cumplir con todas las exigencias impuestas.

La característica emprendedora personal de Autoconfianza e Independencia presentó una diferencia significativa que pudo deberse a que el estudiante, al haber cursado un año más de la carrera, ya siente mayor seguridad gracias a la experiencia adquirida a través de las pruebas, trabajos realizados y técnicas aplicadas en los laboratorios. Las restantes CEP's no registraron diferencias significativas lo cual indica que las modificaciones propuestas 
en la planificación de los Laboratorios de Química Analítica I y Química Analítica II, que incluyeron al ABP y AC, no influyeron en el fortalecimiento de estas o que las actividades no fueron suficientes para lograr este objetivo.

Con respecto a la línea de investigación para trabajos futuros se prevé la incorporación de otros instrumentos de evaluación, con el fin de minimizar la influencia de la subjetividad del cuestionario de autoevaluación, el ajuste de la planificación para que más actividades puedan ser desarrolladas con las metodologías del $\mathrm{ABP}$ y $\mathrm{AC}$ en el laboratorio, de modo a que las ventajas de su utilización suponen la influencia positiva sobre las CEP's y la valoración cualitativa de los resultados finales de parte de los estudiantes para evaluar la conformidad con los mismos.

\section{LISTA DE REFERENCIAS}

Briasco, I. (2014). El desafío de emprender en el siglo XXI. Herramientas para desarrollar la competencia emprendedora. Narcea.

FAUTAPO, P. d. (2010). Módulo de Formación para el Espíritu Emprendedor.

FCQ UNA, F. d. (20 de 12 de 2020). Recuperado el 24 de Noviembre de 2017, de Facultad de Ciencias Químicas: https://qui.una.py/academico/ciencia-y-tecnologia-dealimentos/perfil-del-egresado-2/

FCQ UNA, F. d. (20 de 12 de 2020). Recuperado el 24 de Noviembre de 2017, de Facultad de Ciencias Químicas: https://qui.una.py/academico/quimica-industrial/perfildel-egresado/

OEI, O. d. (2010). Metas Educativas 2012, La Educación que queremos para la generación de los bicentenarios (Primera ed.). Madrid: Cudipal.

TEC de Monterrey. (s.f.). Aprendizaje Colaborativo. Técnicas Didácticas. 